\title{
Relationship between pulmonary function and lung cancer surgical outcome
}

\author{
T. Win*, A. Jackson\#, L. Sharples ${ }^{*}$, A.M. Groves ${ }^{+}$, F.C. Wells ${ }^{\S}$, \\ A.J. Ritchie ${ }^{\S}$ and C.M. Laroche*
}

ABSTRACT: The British Thoracic Society and American College of Chest Physician guidelines outline criteria for investigating patients for lung cancer surgery. However, the guidelines are based on relatively old studies. Therefore, the relationship between pulmonary function test results and surgical outcome were studied prospectively in a large cohort of lung cancer patients.

From January 2001 to December 2003, 110 patients underwent surgery for lung cancer. All underwent full lung function testing in order to predict post-operative lung function.

The hospital mortality rate was $3 \%$ and major complication rate $22 \%$. There was poor overall outcome in $13 \%$. Mean pre-operative lung function values were: forced expiratory volume in one second (FEV 1$) 2.0 \mathrm{~L}$ (79.4\% of the predicted value), and carbon monoxide diffusing capacity of the lung $(D L, C O)$ 73.6\% pred. The mean post-operative lung function values were: FEV 1 1.4 $L(55.6 \%$ pred), and DL,CO $51.3 \%$ pred. All lung function values were better predictors of poor surgical outcome when expressed as a percentage of the predicted value. Using a threshold of preoperative $\mathrm{FEV}_{1}$ of $47 \%$ pred resulted in the most useful positive and negative predictive probabilities, 0.90 and 0.67 , respectively.

Lung function values expressed as a percentage of the predicted value are more useful predictors of post-operative outcome than absolute values. The threshold of predicted forced expiratory volume in one second for surgical intervention could be lower (45-50\% pred) than is currently accepted without increased mortality.

\section{KEYWORDS: Lung carcinoma, lung function, lung function tests, surgical resection}

urgical resection remains the treatment of choice for anatomically resectable nonsmall cell lung cancer [1, 2], offering the best prospect of long-term survival. However, many patients have coexisting chronic airflow limitation [3], which is associated with an increased risk from surgery. Loss of lung tissue may grossly impair post-operative ventilatory function in such patients, predisposing to cardiopulmonary complications, including death. The British Thoracic Society (BTS) and American College of Chest Physician guidelines [4, 5] outline criteria for investigating patients with borderline lung function. However, many of the studies on which the guidelines are based are relatively old, and often based on predominantly male patients who were significantly younger than patients currently being considered for surgery.

The latest BTS guidelines suggest that further investigation is unnecessary if a patient has a forced expiratory volume in one second (FEV1) of $>2.0 \mathrm{~L}$ for pneumonectomy or $>1.5 \mathrm{~L}$ for lobectomy. This is because studies have shown a mortality rate of $<5 \%$ using these criteria. However, these figures are based mainly on studies involving males, and it is likely that lower values would be more appropriate for females. It is possible that a figure based on a value expressed as a percentage of the predicted value would be better still, since this would also take into account the patient's age, sex and height. At present, if spirometry shows the FEV1 to be less than the values given above, then the BTS guidelines recommend a sequence of investigations, consisting of ventilation/ perfusion $\left(V^{\prime} / Q^{\prime}\right)$ scintigraphy in order to estimate the post-operative FEV1 (ppo FEV1) and carbon monoxide diffusing capacity of the lung ( $D \mathrm{~L}, \mathrm{CO} ;$ ppo $\mathrm{DL}, \mathrm{CO})$ as a percentage of the predicted value. This is followed by an exercise test, either a shuttle walking test or a formal cardiopulmonary exercise test.

\section{AFFILIATIONS}

*Thoracic Oncology, and Depts of \#Respiratory Physiology, and

${ }^{\S}$ Cardiothoracic Surgery, Papworth Hospital, and

- Medical Research Council (UK) Biostatistics Unit, and

${ }^{+}$Dept of Radiology and Nuclear Medicine, Addenbrooke's Hospital, Cambridge, UK

CORRESPONDENCE

T. Win

Thoracic Oncology Unit

Papworth Hospital

Papworth Everard

Cambridge

CB3 8RE

UK

Fax: 441480364331

E-mail: thida.win@papworth.nhs.uk

Received:

June 282004

Accepted after revision:

November 262004

SUPPORT STATEMENT

T. Win received a National Health Service Eastern Region R\&D Office (Cambridge, UK) Research and Development Grant. 
Many previous studies have shown predictive values for specific cut-off levels of FEV1 and DL,CO [6-11]. A study performed by PIERCE et al. [12] suggested that the product of ppo FEV1 and ppo DL,CO (both \% pred) was the most useful predictor of survival from surgery. This value, termed the predicted post-operative product (PPP), incorporates baseline assessments of FEV1, DL,CO, perfusion scans and the proportion of lung to be resected in a single index. The benefit of the PPP is that it prevents patients being turned down on the basis of a single criterion. It would also be potentially applicable to both males and females, since it depends on the use of percentage predicted rather than absolute values for FEV1 and DL,CO. However, the original study by PIERCE et al. [12] was relatively small (54 patients) and only included males. Further evaluation in a larger study including females was necessary in order to determine the wider applicability of the PPP, and to confirm the specific relationships between the PPP and probability of survival from surgery.

Therefore, the relationship between pulmonary function test results and surgical outcome were prospectively studied in a reasonably large cohort of currently typical resectable lung cancer patients at the cardiothoracic centre of Papworth Hospital (Cambridge, UK). In particular, the aim was to establish which of the following measurements best predicted poor surgical outcome: 1) pre-operative absolute FEV1; 2) preoperative FEV1 (\% pred); 3) pre-operative DL,CO (\% pred); 4) ppo absolute FEV1; 5) ppo FEV1 (\% pred); 6) ppo DL,CO (\% pred); and 7) PPP. The aim was also to establish the lowest safe FEV1 and DL,CO for lung cancer operation.

\section{MATERIALS AND METHODS Study subjects}

Between January 2001 and December 2003, 150 patients were assessed for curative surgery for lung cancer at Papworth Hospital. Of these, 32 were not referred for surgery and were excluded from the study. A further eight patients were deemed not resectable at the time of exploration (open and closed) and were not studied further. The remaining 110 patients made up the study sample. Written informed consent was given by all patients and the Huntington Ethics Committee (Fulbourn, UK) approved the study.

All patients underwent full lung function testing. Thirty-two $(29 \%)$ showed borderline lung function (FEV1 $<1.5 \mathrm{~L}$ for lobectomy and $<2.0 \mathrm{~L}$ for pneumonectomy).

The patients also underwent perfusion scintigraphy in order to predict post-operative lung function. Formal cardiopulmonary exercise tests were performed on 105 patients in order to determine maximal oxygen uptake $\left(V^{\prime} \mathrm{O}_{2}, \max \right)$ and, thus, predict operability. In order to determine the lowest safe operable lung function values, a lower threshold value of $V^{\prime} \mathrm{O}_{2}$, $\max$ of $10 \mathrm{~mL} \cdot \mathrm{kg}$ body weight ${ }^{-1} \cdot \mathrm{min}^{-1}$ was used. However, the final decision as regards operability was made by the surgeon.

\section{Outcome assessment}

The duration of hospital stay and outcome of surgery, including complication and mortality rates, were documented. The in-patient hospital stay was divided into periods of time in intensive care and time on the surgical ward. A complicated post-operative course was defined by the occurrence of any of the following: post-operative death, nonfatal myocardial infarction, heart failure, renal failure, respiratory failure, pulmonary embolism, septicaemia or pneumonia. However, since not all of these complications are irreversible or untreatable, not all of them would necessarily have precluded surgery if predicted in advance. Therefore, for the purpose of this analysis, in order to assess the ability of lung function test results to predict poor outcome, a more restricted definition of poor outcome, defined as post-operative death or respiratory failure, was used to identify patients who ideally should have been identified as high risk prior to surgery.

\section{Full lung function test protocol}

Spirometric assessment of patients was performed in the pulmonary function laboratory of Papworth Hospital according to Association of Respiratory Technology and Physiology (1999) guidelines [13]. Patient height was formally assessed and compared with standard normal values [14].

\section{FEV1 and FVC}

The spirometer and recording system were calibrated daily. Measurements recorded for analysis were the best of three reproducible attempts. FEV1 was calculated from a record of forced vital capacity (FVC) performed on a wedge-bellows 12-s Vitalograph spirometer (Vitalograph, Ennis, Ireland).

\section{Carbon monoxide diffusing capacity of the lung}

The DL,CO and diffusion coefficient (DL,CO/alveolar volume) were estimated using the carbon monoxide single-breath technique.

After maximal expiration, the patients were asked to inhale quickly and as deeply as possible. The patient had to achieve $\geqslant 90 \%$ of vital capacity and inspire in $<2$ s. If the patient had an obstruction, the inhalation time was increased to $4 \mathrm{~s}$. The patient held their breath for $10 \mathrm{~s}$ without straining. The test was performed at least twice with a time delay of $4 \mathrm{~min}$ between each attempt. At least two technically acceptable test results were obtained and the mean reported.

\section{Lung volume}

The patient was seated in a whole-body plethysmograph box (Masterscreen; Viasys Healthcare, Warwick, UK) for performing a slow vital capacity manoeuvre by exhaling until residual volume and then inspiring fully to total lung capacity before returning to normal tidal breathing. The test was repeated until three technically acceptable traces were obtained, and the mean of these was reported.

\section{Cardiopulmonary exercise test (treadmill maximal oxygen uptake test)}

The cardiopulmonary exercise test was performed using the Oxycon Pro (Viasys Healthcare) exercise system with the standardised exponential exercise protocol described by NORTHRIDGE et al. [15], adapted to allow an additional 1-min warm-up period. Measurements made during this test included full metabolic gas exchange and ECG. The test lasted for a maximum of $20 \mathrm{~min}$, of which the patient exercised for $16 \mathrm{~min}$ ( $4 \mathrm{~min}$ are taken up with baseline measurements and recovery). The patient was required to exercise for as long as 
possible until symptom restricted. The ECG was monitored, and oxygen and carbon dioxide in the expired air measured.

\section{Quantitative perfusion scan protocols}

All scintigraphy was performed in Papworth Hospital Nuclear Medicine Dept. An Elscint Apex SP4 single-headed camera (Elscint, Haifa, Israel) was used for the study. Patients were imaged in the erect position. The tests were supervised by qualified nuclear medicine technicians and reported by the chest radiologists.

Technetium-99m macroaggregated albumin (75 MBq) was used for the perfusion studies. An intravenous bolus was injected slowly, during which time the patient breathed deeply. Concurrent perfusion analysis using images for all eight views were interpreted subjectively. Relative perfusion analysis was performed on anterior and posterior images. The scan was reported for regions of interest with regard to relative perfusion of the right and left lungs divided into upper, middle and lower zones.

\section{Predicted post-operative FEV1}

The following equation was used to estimate the predicted post-lobectomy FEV1 using perfusion scan results [16]: predicted ppo FEV1=pre-operative FEV1(1-functional contribution of perfusion of the region to be resected). In order to estimate the predicted post-pneumonectomy FEV1, the equation was as follows: FEV1=pre-operative FEV1(1-percentage perfusion of lung to be resected). The PPP was also calculated [12].

\section{Thoracotomy}

Standard posteriolateral thoracotomy was performed by one of three dedicated cardiothoracic surgeons. The routine surgical and anaesthetic procedure included single-lung ventilation using a double-lumen endobronchial tube during the operation. Standard post-operative physiotherapy was performed, involving breathing exercises and early ambulation.

\section{Statistical analysis}

Lung function measurements are summarised as mean \pm SD for each group. Associations between measures of lung function and outcome groups were assessed using an unpaired t-test. These associations were adjusted for operation type (pneumonectomy/lobectomy) using ANOVA, with outcome as a fixed effect and operation type as a covariate. Receiver-operating characteristic curves were plotted in order to assess the predictive value of lung function test results for poor outcome. Positive and negative predictive values were calculated for selected thresholds and using study estimates of the prevalence of poor outcome.

\section{RESULTS}

All 110 patients completed the study. Of the 110 patients in the study group, $44(40 \%)$ were female and $66(60 \%)$ male. Their mean \pm SD age was $69 \pm 8$ yrs (range $42-85$ yrs). Thirty-six patients (33\%) underwent pneumonectomy and the remaining patients underwent single lobectomy $(59 \%)$, bilateral lobectomy $(4 \%)$ or wedge resection $(4 \%)$. The majority of patients had squamous cell carcinoma (44\%), adenocarcinoma (34\%) or nonsmall cell lung cancer (14\%). Tumours were staged as follows: 1A (40\%), 1B (23\%), 2A (4\%), 2B (17\%), 3A (14\%), and $3 \mathrm{~B}(2 \%)$.

The baseline pulmonary function test results of the study group are recorded in table 1 . The lowest safe operated preoperative FEV1 and DL,CO were $1 \mathrm{~L}$ (37\% pred) and 33\% pred, respectively. The lowest operated post-operative values were ppo FEV1 0.6 L (22\% pred), ppo DL,CO 21\% pred and PPP 560. The mean $V^{\prime} \mathrm{O}_{2}$, max was $18.3 \mathrm{~mL} \cdot \mathrm{kg}$ body weight ${ }^{-1} \cdot \mathrm{min}^{-1}$ (range 10.2-34.5 $\mathrm{mL} \cdot \mathrm{kg}$ body weight ${ }^{-1} \cdot \mathrm{min}^{-1}$ ).

Four $(3 \%)$ patients died within 30 days of lung cancer surgery (1\% lobectomy and $8 \%$ pneumonectomy). Twenty-four $(22 \%)$ patients had a major complication, i.e. overall, $28(25 \%)$ patients were considered to have had a complicated postoperative course. The major complications are recorded in table 2. The most common major complications were pneumonia $(19 \%)$ and respiratory failure $(10 \%)$.

Table 3 summarises the differences in lung function for patients with and without a complicated post-operative course. Pre-operative FEV1, pre-operative $D \mathrm{~L}, \mathrm{CO}$, ppo $\mathrm{DL}, \mathrm{CO}$ and ppo FEV1, all expressed as a percentage of the predicted value, were significantly associated with a complicated postoperative course. These results did not change substantially when adjusted for operation type (data not shown).

Fourteen $(13 \%)$ patients had a poor operative outcome defined by 30 -day mortality or respiratory failure. Table 4 summarises the differences in lung function for patients with satisfactory and poor outcomes. Patients with a poor outcome had a significantly lower pre-operative FEV1 (\% pred), but this was not evident from absolute FEV1 measurements. DL,CO measurements were significantly lower for patients with a poor outcome, and ppo DL,CO (\% pred), ppo FEV1 (\% pred) and PPP were significantly associated with outcome.

Figure 1 shows the receiver-operating characteristic curves. The diagonal line indicates an area under the curve of 0.5 , equivalent to the measurement having no predictive value. The

\begin{tabular}{l|c|}
\hline TABLE 1 & $\begin{array}{l}\text { Pulmonary function data of lung cancer surgery } \\
\text { patients }\end{array}$ \\
\hline FEV $\mathbf{~ L ~}$ & $2.0 \pm 0.7(1.0-4.7)$ \\
FEV 1 \% pred & $79.4 \pm 20.3(37-119)$ \\
FVC L & $3.3 \pm 0.9(2-6)$ \\
FVC \% pred & $101 \pm 17.3(56-155)$ \\
DL,Co \% pred & $73.6 \pm 18.0(33-122)$ \\
DL,Co/VA \% pred & $85.9 \pm 20.4(37-157)$ \\
TLC \% pred & $106.5 \pm 15.2(66-144)$ \\
ppo FEV $\mathbf{1}$ L & $1.4 \pm 0.5(0.6-3.6)$ \\
ppo FEV 1 \% pred & $55.6 \pm 15.4(22-95)$ \\
ppo DL,CO \% pred & $51.3 \pm 14.9(21-92)$ \\
PPP & $2990 \pm 1445(560-8423)$ \\
\hline
\end{tabular}

Data are presented as mean \pm SD (range). FEV1: forced expiratory volume in one second; \% pred: percentage of the predicted value; FVC: forced vital capacity; $\mathrm{LL}, \mathrm{CO}$ : carbon monoxide diffusing capacity of the lung; $V_{A}$ : alveolar volume; TLC: total lung capacity; ppo FEV1: predicted post-operative FEV1; ppo $D \mathrm{~L}, \mathrm{CO}$ : predicted post-operative $\mathrm{DL}, \mathrm{CO}$; PPP: predicted post-operative product (product of ppo FEV 1 and ppo $D \mathrm{~L}, \mathrm{CO}$ (both \% pred)). \#: $\mathrm{n}=110$ 


\begin{tabular}{|c|c|c|}
\hline $\begin{array}{cc}\text { TABLE } 2 & \text { Frequ } \\
& \text { comp } \\
& \text { outco }\end{array}$ & $\begin{array}{l}\text { major complicatic } \\
\text { post-operative col }\end{array}$ & $\begin{array}{l}\text { s comprising } \\
\text { e and poor }\end{array}$ \\
\hline Complication & Complicated course & Poor outcome \\
\hline Subjects $^{\#} \mathbf{n}$ & 28 & 14 \\
\hline Death within 30 days & 4 & 4 \\
\hline Myocardial infarction & 3 & \\
\hline Cardiac failure & 3 & \\
\hline Renal failure & 3 & \\
\hline Respiratory failure & 11 & 11 \\
\hline Sepsis & 3 & \\
\hline Pneumonia & 21 & \\
\hline Pulmonary embolism & 2 & \\
\hline
\end{tabular}

TABLE 3 Pulmonary function data for patients with and without a complicated post-operative course

\begin{tabular}{|c|c|c|c|c|}
\hline & Uncomplicated & Complicated & $\begin{array}{c}\text { Difference } \\
(95 \% \mathrm{Cl})\end{array}$ & p-value \\
\hline Subjects n & 82 & 28 & & \\
\hline $\mathrm{FEV}_{1} \mathrm{~L}$ & $2.0 \pm 0.6$ & $1.9 \pm 0.8$ & $0.1(-0.2-0.4)$ & 0.44 \\
\hline FEV1 \% pred & $82.9 \pm 19.5$ & $69.6 \pm 19.4$ & $13.4(4.9-21.8)$ & 0.002 \\
\hline FVC L & $3.2 \pm 0.8$ & $3.4 \pm 1.0$ & $-0.2(-0.6-0.2)$ & 0.24 \\
\hline FVC \% pred & $102.4 \pm 17.7$ & $96.7 \pm 15.6$ & $5.7(-1.8-13.2)$ & 0.13 \\
\hline DL,Co \% pred & $76.4 \pm 17.9$ & $65.0 \pm 15.9$ & $11.4(3.9-19.1)$ & 0.004 \\
\hline ppo FEV 1 L & $1.4 \pm 0.5$ & $1.3 \pm 0.5$ & $0.1(-0.1-0.3)$ & 0.34 \\
\hline ppo FEV $1 \%$ pred & $58.0 \pm 14.5$ & $49.2 \pm 16.4$ & $8.8(3.3-15.3)$ & 0.009 \\
\hline ppo $D L, C o \%$ pred & $53.8 \pm 14.8$ & $44.8 \pm 13.1$ & $9.0(2.5-15.4)$ & 0.007 \\
\hline PPP & $3228 \pm 1428$ & $2346 \pm 1309$ & $882(257-1507)$ & 0.006 \\
\hline
\end{tabular}

Data are presented as mean $\pm \mathrm{SD}$, unless otherwise stated. $\mathrm{Cl}$ : confidence interval; FEV 1 : forced expiratory volume in one second; \% pred: percentage of the predicted value; FVC: forced vital capacity; DL,CO: carbon monoxide diffusing capacity of the lung; ppo FEV1: predicted post-operative FEV1; ppo $D \mathrm{~L}, \mathrm{CO}$ : predicted post-operative $D \mathrm{~L}, \mathrm{CO}$; PPP: predicted post-operative product (product of ppo FEV1 and ppo DL,CO (both \% pred)).

area under the curve is greater for FEV1 expressed as a percentage of the predicted value than as absolute value pre-operatively $(0.73$ versus 0.59$)$, or as a predicted postoperative value by $V^{\prime} / Q^{\prime}$ scan (0.75 versus 0.59 ; fig. 1$)$. As FEV1 measured in litres has a lesser area under the curve, a threshold was not assessed to predict the outcome further. If pre-operative FEV1 is used to predict the outcome, and assuming a threshold of $65 \%$ for defining high risk for lung cancer surgery, the positive and negative predictive values are 93 and $29 \%$, respectively. That is, the probability of a satisfactory outcome if FEV1 $\geqslant 65 \%$ is 0.93 and the probability of a poor outcome if FEV $1<65 \%$ is 0.29 . The corresponding positive and negative predictive probabilities for a ppo FEV1 of $<40 \%$ were 90 and $33 \%$, respectively. If a new threshold of preoperative FEV1 of $47 \%$ is used to define a high risk for lung
TABLE 4 Pulmonary function data for patients with and without a poor outcome

\begin{tabular}{lcccc} 
& $\begin{array}{c}\text { Satisfactory } \\
\text { outcome }\end{array}$ & $\begin{array}{c}\text { Poor } \\
\text { outcome }\end{array}$ & $\begin{array}{c}\text { Difference } \\
(\mathbf{9 5 \%} \mathbf{C l})\end{array}$ & p-value \\
\hline Subjects n & 96 & 14 & & \\
FEV $\mathbf{1}$ & $2.0 \pm 0.6$ & $1.9 \pm 0.9$ & $0.2(-0.2-0.5)$ & 0.42 \\
FEV 1 \% pred & $81.8 \pm 19.5$ & $63.0 \pm 18.4$ & $18.8(7.8-29.8)$ & 0.001 \\
FVC L & $3.2 \pm 0.8$ & $3.6 \pm 1.1$ & $-0.3(-0.8-0.2)$ & 0.18 \\
FVC \% pred & $102.0 \pm 17.9$ & $93.6 \pm 10.3$ & $8.4(-1.3-18.1)$ & 0.01 \\
DL,CO \% pred & $75.7 \pm 17.1$ & $59.7 \pm 17.7$ & $15.9(6.2-25.7)$ & 0.002 \\
ppo FEV $\mathbf{1}$ L & $1.4 \pm 0.5$ & $1 . \pm 0.5$ & $0.2(-0.1-0.5)$ & 0.12 \\
ppo FEV 1 \% pred & $57.7 \pm 14.7$ & $42.0 \pm 13.0$ & $15.7(7.5-24.0)$ & $<0.001$ \\
ppo DL,CO \% pred & $53.5 \pm 14.6$ & $37.9 \pm 8.3$ & $15.6(7.6-23.5)$ & $<0.001$ \\
PPP & $3209 \pm 1417$ & $1644 \pm 705$ & $1564(796-2333)$ & $<0.001$ \\
\hline
\end{tabular}

Data are presented as mean $\pm \mathrm{SD}$, unless otherwise stated. $\mathrm{Cl}$ : confidence interval; FEV1: forced expiratory volume in one second; \% pred: percentage of the predicted value; FVC: forced vital capacity; $D L, C O$ : carbon monoxide diffusing capacity of the lung; ppo FEV1: predicted post-operative FEV1; ppo $D \mathrm{~L}, \mathrm{CO}$ : predicted post-operative $D \mathrm{~L}, \mathrm{CO}$; PPP: predicted post-operative product (product of ppo FEV 1 and ppo DL,CO (both \% pred)).

cancer surgery, the probability of a satisfactory outcome if FEV1 $>47 \%$ is $0.90(94 / 104)$ and the probability of a poor outcome if FEV1 $\leqslant 47 \%$ is $0.67(4 / 6)$.

The area under the curve for pre-operative $D \mathrm{~L}, \mathrm{CO}$ (\% pred) was 0.70 and for ppo DL,CO (\% pred) was 0.77 (fig. 1). For preoperative $D \mathrm{~L}, \mathrm{CO}$ (\% pred), the probability of a satisfactory outcome if $D \mathrm{~L}, \mathrm{CO} \geqslant 45 \%$ is $0.90(93 / 103)$ and the probability of

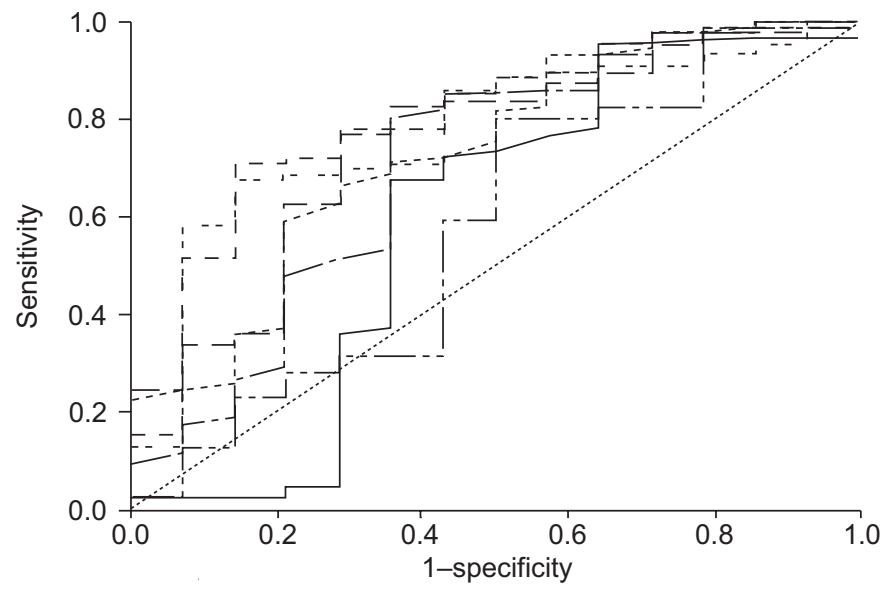

FIGURE 1. Receiver-operating characteristic curves (- - baseline forced expiratory volume in one second (FEV1; L; area under the curve (AUC) 0.587); ------: baseline FEV1 (\% pred; AUC 0.731); _ _ _ : baseline carbon monoxide diffusing capacity of the lung ( $D \mathrm{~L}, \mathrm{CO}$; \% pred; AUC 0.701); — - - - : baseline predicted postoperative (ppo) FEV1 (L; by ventilation/perfusion ( $\left.V^{\prime} / Q^{\prime}\right)$ scan; AUC 0.587); -- --: baseline ppo $D$ L,CO (\% pred; by $V^{\prime} / Q^{\prime}$ scan; AUC 0.772); - - -: predicted postoperative product (PPP; AUC 0.792); — —: baseline ppo FEV1 (\% pred; by $V^{\prime} / Q^{\prime}$ scan; AUC 0.752); …..... reference line (AUC 0.5). Diagonal segments are produced by ties. The PPP is the product of ppo FEV 1 and ppo DL,CO (both \% pred) 
a poor outcome if $D \mathrm{~L}, \mathrm{CO}<45 \%$ is 0.57 (4/7). Neither ppo FEV1 nor ppo $D L, C O$ is better than the pre-operative value in predicting outcome (data not shown).

The PPP exhibited the greatest area under the curve at 0.79 (fig. 1). If PPP is used to predict outcome, and assuming a threshold of 1,600 for defining a high risk for lung cancer surgery, the probability of a satisfactory outcome if PPP $\geqslant 1,600$ is $0.90(87 / 96)$ and the probability of a poor outcome if PPP $<1,600$ is 0.35 (5/14). A new threshold of 760 results in the probability of a satisfactory outcome if PPP $\geqslant 760$ of 0.89 $(95 / 106)$ and of a poor outcome if PPP $<760$ of $0.75(3 / 4)$.

\section{DISCUSSION}

Surgical resection remains the curative treatment of choice in patients with lung cancer. However, it has proved difficult to assess the lower limit of surgical tolerance. Several attempts have been made to determine these physiological limits $[9,17]$. Previous studies have shown predictive values for specific cut-off levels of baseline test results. Some have reported absolute values and others percentage predicted values. Three studies suggested that, if the pre-operative FEV1 is $>1.5 \mathrm{~L}$ for lobectomy and $>2.0 \mathrm{~L}$ for pneumonectomy, the mortality rates should be $<5 \%[6,18,19]$. Alternatively, PUTNAM et al. [20] suggested that a pre-operative FEV1 of $<65 \%$ pred is a significant risk factor.

There is substantial evidence that operative risk is related to absolute ppo FEV1 [6-8, 12, 21-23]. As a result of the findings of these investigators, it was recommended that a ppo FEV1 of $>0.8 \mathrm{~L}$ is used as the cut-off for surgery. Others have found it more useful to use percentage predicted FEV1, recommending a threshold of $>50 \%$ pred for FEV1 [10] or $>40 \%$ pred for ppo FEV1 [9]. For the latter, a value of $<40 \%$ was associated with $50 \%$ mortality. They also found a ppo $D \mathrm{~L}, \mathrm{CO}$ of $<40 \%$ pred was a good predictor of post-operative death. Another study recommended a lower cut-off threshold of $34 \%$ pred for ppo FEV1 [20].

In the present study, it was found that the absolute values (preoperative FEV1, FVC and ppo FEV1) did not predict surgical outcome defined by 30-day mortality and post-operative respiratory failure. In contrast, all the values corrected for age and sex (i.e. pre-operative $\mathrm{FEV} 1$, pre-operative $\mathrm{DL}, \mathrm{CO}$, ppo FEV1, ppo DL,CO and PPP, all expressed as a percentage of the normal predicted value) correlated significantly with both complicated post-operative course and poor surgical outcome. These findings reflect the fact that previous studies included a more homogeneous surgical population, consisting almost entirely of male patients of relatively young age. Since current surgical lung cancer patients are more likely to be elderly and/ or female, it is no longer appropriate to use absolute lung function values. As such, future guidelines should adopt percentage predicted rather than absolute values.

In the present patients, using an FEV1 threshold of $65 \%$ pred, there was good positive prediction in that $93 \%$ of patients with values above this threshold had a satisfactory outcome from surgery. However, $71 \%$ of patients with values below this threshold also had a satisfactory outcome, suggesting that it may be too high. Similar predictive values were found for ppo FEV1 (\% pred). Therefore, lower thresholds were established for pre-operative FEV1 (\% pred), pre-operative DL,CO (\% pred) and PPP that were optimum for the present sample. The thresholds of $47 \%$ pred for pre-operative FEV1, $45 \%$ pred for pre-operative DL,CO and 760 for PPP were optimum. It should be emphasised that these thresholds were established using retrospective analysis. It was found that the lowest safe preoperative FEV1 was lower than previously reported. The absolute value of pre-operative FEV1 in the present sample was $1 \mathrm{~L}$ (37\% pred) and for pre-operative DL,CO was 33\% pred. The lowest post-operative values (ppo FEV1 $0.6 \mathrm{~L}$ (22\% pred), ppo DL,CO $21 \%$ pred and PPP 560) were also much lower than the recommended levels. Despite this, the present 30-day mortality rate ( $3 \%$ for all thoracotomy, $1 \%$ for lobectomy and $8 \%$ for pneumonectomy) was lower than the BTS guidelines for lobectomy $(4 \%)$, and in line with BTS guidelines for pneumonectomy $(8 \%)$ and the open and closed rate (5-10\%). This reflects recent improvements in staging and surgical techniques and perioperative care, and results should be interpreted in this context. The present authors would not routinely recommend such low cut-off values without patients undergoing extensive fitness assessment, discussion at multidisciplinary meetings and surgeons that have the necessary expertise for dealing with difficult cases.

The present study has limitations in that, although it was a relatively large series, some of the negative predictive values were based on low numbers and were measured relatively imprecisely. Use of these thresholds should be tested in further larger series. In addition, the definition of poor outcome was limited to short-term post-surgery indices, and longer-term follow-up would be needed to confirm the longer-term predictive value of these cut-off values of lung function.

Since it was found that, with vigorous investigation, lower thresholds could be safely applied, it may be necessary to reconsider the surgical assessment guidelines for lung cancer patients. This would require close liaison between the referring chest physician and operating surgeons. If the present recommendations are confirmed in longer follow-up studies, such measures would hopefully improve surgical operation rates, cure rates and, hence, lung cancer mortality. For these reasons, using lower lung function thresholds would be beneficial.

In conclusion, the present authors recommend that percentage predicted rather than absolute lung function values be used in assessing patients for lung cancer surgery. The lower threshold of forced expiratory volume in one second for surgical intervention could be lower than is currently suggested, namely $45-50 \%$ of the predicted value, without increased mortality.

\section{REFERENCES}

1 Pearson FG. Current status of surgical resection for lung cancer. Chest 1994; 106: Suppl. 6, 337S-339S.

2 Reif MS, Socinski MA, Rivera MP. Evidence-based medicine in the treatment of non-small-cell lung cancer. Clin Chest Med 2000; 21: 107-120.

3 Legge JS, Palmer KN. Pulmonary function in bronchial carcinoma. Thorax 1973; 28: 588-591.

4 British Thoracic Society, Society of Cardiothoracic Surgeons of Great Britain and Ireland Working Party. BTS guidelines: guidelines on the selection of patients with lung cancer for surgery. Thorax 2001; 56: 89-108. 
5 Beckles MA, Spiro SG, Colice GL, Rudd RM, American College of Chest Physicians. The physiologic evaluation of patients with lung cancer being considered for resectional surgery. Chest 2003; 123: Suppl. 1, 105S-114S.

6 Boushy SF, Billig DM, North LB, Helgason AH. Clinical course related to preoperative and postoperative pulmonary function in patients with bronchogenic carcinoma. Chest 1971; 59: 383-391.

7 Boysen PG, Block AJ, Olsen GN, Moulder PV, Harris JO, Rawitscher RE. Prospective evaluation for pneumonectomy using the ${ }^{99 \mathrm{~m}}$ technetium quantitative perfusion lung scan. Chest 1977; 72: 422-425.

8 Boysen PG, Harris JO, Block AJ, Olsen GN. Prospective evaluation for pneumonectomy using perfusion scanning: follow-up beyond one year. Chest 1981; 80: 163-166.

9 Markos J, Mullan BP, Hillman DR, et al. Preoperative assessment as a predictor of mortality and morbidity after lung resection. Am Rev Respir Dis 1989; 139: 902-910.

10 Nakahara K, Monden Y, Ohno K, Miyoshi S, Maeda H, Kawashima Y. A method for predicting postoperative lung function and its relation to postoperative complications in patients with lung cancer. Ann Thorac Surg 1985; 39: 260-265.

11 Nakahara K, Ohno K, Hashimoto J, et al. Prediction of postoperative respiratory failure in patients undergoing lung resection for lung cancer. Ann Thorac Surg 1988; 46: 549-552.

12 Pierce RJ, Copland JM, Sharpe K, Barter CE. Preoperative risk evaluation for lung cancer resection: predicted postoperative product as a predictor of surgical mortality. Am J Respir Crit Care Med 1994; 150: 947-955.

13 Hill S, Newall C, eds. Practical Handbook of Respiratory Function Testing. Stoke-on-Trent, Association of Respiratory Technology and Physiology, 1999.

14 Cotes JE, Dabbs JM, Hall AM, Heywood C, Laurence KM. Sitting height, fat-free mass and body fat as reference variables for lung function in healthy British children: comparison with stature. Ann Hum Biol 1979; 6: 307-314.

15 Northridge DB, Grant S, Ford I, et al. Novel exercise protocol suitable for use on a treadmill or a bicycle ergometer. Br Heart J 1990; 64: 313-316.

16 Bolliger CT, Guckel C, Engel $\mathrm{H}$, et al. Prediction of functional reserves after lung resection: comparison between quantitative computed tomography, scintigraphy, and anatomy. Respiration 2002; 69: 482-489.

17 Ferguson MK, Little L, Rizzo L, et al. Diffusing capacity predicts morbidity and mortality after pulmonary resection. J Thorac Cardiovasc Surg 1988; 96: 894-900.

18 Miller JI Jr. Physiologic evaluation of pulmonary function in the candidate for lung resection. J Thorac Cardiovasc Surg 1993; 105: 347-351.

19 Wernly JA, DeMeester TR, Kirchner PT, Myerowitz PD, Oxford DE, Golomb HM. Clinical value of quantitative ventilation-perfusion lung scans in the surgical management of bronchogenic carcinoma. J Thorac Cardiovasc Surg 1980; 80: 535-543.

20 Putnam JB Jr, Lammermeier DE, Colon R, McMurtrey MJ, Ali MK, Roth JA. Predicted pulmonary function and survival after pneumonectomy for primary lung carcinoma. Ann Thorac Surg 1990; 49: 909-914.

21 Olsen GN, Block AJ, Swenson EW, Castle JR, Wynne JW. Pulmonary function evaluation of the lung resection candidate: a prospective study. Am Rev Respir Dis 1975; 111: 379-387.

22 Pate P, Tenholder MF, Griffin JP, Eastridge CE, Weiman DS. Preoperative assessment of the high-risk patient for lung resection. Ann Thorac Surg 1996; 61: 1494-1500.

23 Kearney DJ, Lee TH, Reilly JJ, DeCamp MM, Sugarbaker DJ. Assessment of operative risk in patients undergoing lung resection. Importance of predicted pulmonary function. Chest 1994; 105: 753-759. 